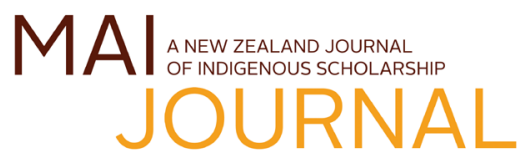

DOI: 10.20507/MAlJournal.2019.8.3.3

\title{
HEI MATAAHO
}

\section{He tuhinga Kaupapa Māori}

\author{
Krystal Te Rina Warren* \\ Kewa Cashell-Warren ${ }^{\dagger}$
}

\section{He whakarāpopoto}

Kei te puta te tokomaha o ngāi raukura (ngā tauira o mua o te Kura Kaupapa Māori) ki ngā whare wānanga puta noa i te ao, heoi anō, he ruarua noa ngā tuhinga reo Māori e arotake ana i te Kaupapa Māori. E mātai ana tēnei tuhinga i te ahunga mai o te Kura Kaupapa Māori hei tauranga ātete i ngā tāmitanga a tauiwi. He kōrero tēnei mō te ariā Kaupapa Māori (mā ngā tauira whare wānanga): he tautoko $i$ te putanga o te tuhinga taiea, te tuhinga $\mathrm{i}$ ăta rangahaua, te tuhinga arohaehae, te tuhinga reo Māori. Ko te tikanga ia, ka puta he tuhinga mō ngā kaupapa huhua, engari ka taka te tuhinga nei i te whakaaro mō te mātātuhi wewete mō ngā raukura. Kāti, mā tēnei tuhinga te mautohe, te whakarauora me te pupuri i te reo Māori, te mātauranga Māori, me te wairua Māori, e whakanui.

\section{He kupu matua}

Kaupapa Māori, Kura Kaupapa Māori

\section{Hei mataaho: He tuhinga Kaupapa Māori}

Tēnei au te ira nō tuawhakarere,

Ko te ira Atua, tēnei au

He kōnehunehu nō te kore, nō te pō,

Nō te marae ātea, āio

Ko te ira atua, ko te ira wahine

Ko te ira tāne, tēnei au

Nā Pāpā Rawiri Wright (2009) ēnei kupu waiata i tito. He kupu e whakaū ana i te mōhiotanga o te Māori ki tōna orokohanga. He mea kapo atu ènei kupu hei whakataki i te kaupapa tuhituhi nei. Tēnā, me mihi atu ki a Ranginui e tū iho nei, ki a Papatūānuku e takoto ake nei, haumi e, hui e, tāiki e!
Nō te taenga mai o te Pākehā, i noho tonu te mana whakahaere a te Māori ki Aotearoa whānui. Engari, nā te taiāniwhaniwha mai a te manene, a te iwi Pākehā, kua nunumi atu ngā āhuatanga Māori, pērā i te mōhiotanga ko te tangata he ira nō tuawhakarere. He nui ngā pānga o te tāmi ki ngā āhuatanga Māori, ki te rirohanga atu o ngā mōhiotanga Māori me te mana Māori motuhake (Walker, 1990). Ko tētahi o ngā kaiwhakahaere o te tāmitanga ko te kura Pākehā, me ōna uara Pākehā. Kua ātete, kua whakanau, kua mautohe, kua whawhai te iwi Māori ki te tāmi a te Pākehā (Walker, 1984). Ko tētahi o aua tauranga ātete, ko ngā Kura Kaupapa Māori. Heoi anō, i te tau 2019, kei te whare wānanga ngà raukura o Te Aho Matua me ngā Kura Kaupapa Māori, ā, he ruarua

* Rangitāne, Matakore-Rangatahi (Tainui), Whitikaupeka (Mōkai Pātea). Te Kunenga ki Pūrehuroa (Massey University), Senior Lecturer, Toikura, Te Pūtahi-a-Toi, Palmerston North. MA (Development Studies).k.t.warren@massey.ac.nz

† Tūwharetoa, Kahungunu, Ngāi Tāmanuhiri. Te Kunenga ki Pūrehuroa (Massey University), Tauira o Te Aho Paerewa (Tohu Whakangungu Kaiako). BA (Māori \& Indigenous Studies). 
noa iho ngā tuhinga reo Māori. Āe, he tuhinga o mua pērā i ngā pukapuka a te Kōti Whenua (nō ngā tau 1865-1975 ēnei Pukapuka Meneti o te Kōti Whenua Māori), ngā Niupepa Māori (The New Zealand Digital Library, n.d), ā, kei te whakaputaina he kōrero anō (pērā i a: Poutu, 2014; Mataamua, 2017; Ihimaera \& Kelly, 2017). Engari, he whāiti rawa te puna o ngā tuhinga reo Māori e hāngai ana ki te ahunga mai me te take o te orokohanga o ngā Kura Kaupapa Māori (Cashell-Warren, 2019).

Tēnā, ka mātai atu tēnei tuhinga ki te ahunga o te Kura Kaupapa Māori hei tauranga ātete (Wright, 2018), me te hononga ki te ariā Kaupapa Māori (Kaupapa Māori theory). Heoi anō, ka arotake tēnei tuhinga i te iti o te kōrero i roto i te reo Māori e pā ana ki ēnei kaupapa (CashellWarren, 2019). Kua puta tēnei whakaohorere i te rapu i ngā kōrero mō Te Aho Matua. Nā, ka aro atu tēnei tuhinga ki te nawe a te raukura o Te Aho Matua, ahakoa kei te Whare Wānanga ètahi o rātou ināianei, kei te tūrukiruki tonu ngā kōrero e pā ana ki te Kaupapa Māori i te reo Māori. Nō reira, he aha tētahi huarahi e anga whakamua ai tēnei kaupapa, me ngā āhuatanga o te reo Māori?

\section{Ngā kura Pākehā}

Tau mai rā ki te papa tipua (Wright, 2009)

Nā te hiahia o te iwi Māori ki te whai mātauranga, ka tukuna ngā tamariki Māori ki tāwāhi, ki Poihākena kia kuraina (Barrington \& Beaglehole, 1974)). I whakatūria ngā Kura Mihinare (Mission Schools) i te tau 1816 ki te whai i te mātauranga o te Pākehā (Simon, Tuhiwai-Smith \& Cram, 2001). Mai i taua wā ki ènei rā, kua noho tangata whenua te kura Pākehā hei huarahi tāmi i te Māori. Me te mea hoki, kīhai i noho motuhake tēnei tukanga tāmi ki te Māori. I ngā whenua puta noa i te ao, kua whakamahia te kura Pākehā hei tauranga tāmi i ngā iwi taketake (Fournier \& Crey, 1997), hei whakapākehā i te iwi, hei whakamōtītī anō hoki. I te tau 1844, i puta ko te Native Trust Ordinance ki te tuku pūtea me te whenua Māori ki ngā Kura Mihinare. I te tau 1847, i tū te Education Ordinance ki te whakaū i ngā akoranga whakapono Pākehā, ngā akoranga ahumahi, waihoki, ki te ako i te reo Pākehā. E ai ki a Tomlins-Jahnke rāua ko Warren (2011), he pānga nui tēnei ki te ngarohanga o te reo Māori. Nā te 1858 Native Schools Act i whakaritea he pūtea ki te tuku i ngā tamariki Māori ki ngā kura noho. Ko te whāinga matua o tēnei ture, ko te tango i te tamaiti i tōna kāinga ao Māori, kia tere ake te whakapākehā i a ia (Simon et al., 2001); kia puta te tama hei ringa ahuwhenua, kia puta te kōtiro hei whaiāipo hāwini. Arā, me noho kaimahi te iwi Māori ki te Pākehā.

I āhua moe aua kura i ngā tau o ngā pakanga whenua, engari i te tau 1867 , ka whakaritea te Native Schools Act. Ko te hua o taua Ture, ka tukuna ngā kawenga katoa o ngā kura ki te Māori; ki te hiahia tētahi rohe, iwi rānei ki tētahi kura, ka tukuna e taua iwi tētahi reta ki te Pāremata hei tono i te kura, i te pūtea whakatū, me te pūtea whakahaere kura. Arā, mā te Māori te utu o te kaiako me te whenua e tuku. I te tau 1877 , ka whakatūria te Education Act hei whakarite i ngā kura Pākehā mō ngā tamariki katoa o Aotearoa, Māori mai, Pākehā mai. Ka mutu, nā te Native Schools Code 1880 te whakatau kia kaua te reo Māori e kōrerohia i ngā kura katoa. Ko te whāinga o te Ture rā, he whakapāhunu i te reo Māori, me te whāngai i ngā tikanga a te Pākehā ki ngā tamariki.

E tika ana ngā kōrero a Ahorangi Huia TomlinsJahnke, "ko te kura he kaiwhakahaere matua mō te tikanga ā-iwi, te whakaaro $\bar{a}$-iwi, me te whakatuanui ā-mātauranga" (kua whakamāorihia ngā kupu Pākehā: Tomlins-Jahnke, 2006, wh. 4). Kei te tautoko hoki a Apple (1996) i tēnei whakaaro; e mea ana ia he tauranga te kura i whakatūria hei whangai i te ahurea me ngā tikanga a te Pākehā. I te tau 1907, ka puta te Tohunga Suppression Act e whakataihara ana i ngā mahi a ngā tohunga Māori, arā, ko te tuku ihotanga o ngā mōhiotanga Māori. He ture aukati i te puna mātauranga o te Māori, he ture whakamoe i ngā whare wānanga o te Māori. I ènei rā, kei te rongo tonu i ngā hua o ènei mahi ā-ture a te Pākehā me ngā pānga o te mahi tārua tikanga Pākehā i roto i ngā kura. Nā tēnei, ka patua rawatia te tuakiri o te Māori. Engari, he aha i noho mana ai ēnei momo mahi?

I te tau 1840 , ko te reo Māori te reo matua i Aotearoa. E ai ki ngā tatauranga, i whakapaetia he 250,000 (nui atu rānei) ngā Māori, he āhua 2,000 noa iho ngā Pākehā (Papps, 1985; Pool, 1991). He pānga nui tō te mate Pākehā ki te Māori; i tere mate te Māori. Nō te tau 1852 (te tau i whakaritea te NZ Constitution Act), i heke te tokomaha o ngā Māori ki te 100,000, engari i piki te tokomaha o ngā Pākehā ki te 52,000 tāngata (Papps, 1985; Pool, 1991). Ko tēnei te wā i riro atu ai ngā kawenga a te Karauna mō te wāhi ki Te Tiriti o Waitangi ki te manene Pākehā, ā, i whakatūria te Pāremata Pākehā (Settler Parliament) ki Aotearoa. Haere nei te wā, ka taiāniwhaniwha mai a tauiwi.

Nō te tau 1896, kua heke te tokomaha o ngāi Māori ki te 42,000 (Papps, 1985), ā, kua piki te tokomaha o ngāi Pākehā ki te 750,000 (Von 
Dadelszen, 1901). Nā te huringa o te tatauranga tangata, kua huri hoki te reo e kōrerohia whānuitia ana i te motu, kua huri hoki te mana whakahaere o te motu.

I te tīmatanga o te rautau 1900 , $\mathrm{i}$ arohia te whakaako āhuatanga Māori ki runga i te marae e ngā rangatira o te wā, e Te Pūea Herangi rāua ko Tā Apirana Ngata (King, 1988). Nā te whakakorenga o ngā āhuatanga Māori i ngā kura o te motu, i ngā tau 1930, i tīmata a Te Pūea ki te kuhu i te reo Māori me ōna tikanga ki te marau ako o te motu (King, 1977). Nō muri mai i taua wā, kua aro ngā kaupapa here o te Kāwanatanga ki te whakapākehā i ngā tamariki Māori. I ngā tau 1950 (whai muri i ngā Pakanga o te Ao), i huri tēnei aronga ki te 'whakakotahi' kē. Hei āpititanga ki tēnei, i te tau 1950, i whakatūria ètahi komiti mātauranga (ko te National Advisory Committee on Māori Education tētahi arā, ko NACME)) ka puta hoki ētahi ripoata e pā ana ki te whakaako tamariki Māori (1961 Ripoata a Hunn, me te 1962 Ripoata a te Kāhui o Currie). I tēnei wā i rapua hoki ngā kaiako Māori (Walker, 2016), ā, he nui te manene Māori ki ngā tāone. Ko te whakatau a ngā ripoata, nō ngā tamariki Māori me ō rātou mātua te hē i rahua ai ngā tamariki i ngā kura. Tau mai ki te tau 1970, i puta te Ripoata a NACME e kī ana me uru te reo Māori me ōna tikanga ki te marau (NACME, 1970).

Ahakoa te āhuatanga o te iwi Māori i roto i ngā tau, kīhai i mutu te whawhai a ngā tūpuna mō te mana motuhake me te tino rangatiratanga o te iwi Māori. Nō te Whakaminenga o ngā Hapu Katoa o Niu Tireni (1835) me te Tiriti o Waitangi (1840), ki te Kīngitanga me ngā pakanga whenua (i ngā tau 1860-1880), te Pāremata Māori, Te Hokowhitu-a-Tū, Te Rōpū Wāhine Toko i te Ora, ki ngā porotete o ngā 1970, kāore tēnei mea te mautohe ki ngā mahi tāmi a te Pākehā i mutu (Walker, 1984).

\section{Kura Kaupapa Māori}

Whakarerea ngā kaka taumaha (Wright, 2009)

I rangahaua te oranga o te reo Māori e Richard Benton i ngā tau 1970 (Benton, 1972). I kitea i tōna rangahau, kua heke te tatauranga arero reo Māori; i rangona te māuiui o te reo Māori. Ki te puretumu i te mate i kitea i te rangahau a Benton, ka puta mai ètahi rōpū rangatahi Māori ki te kawe i tēnei take ki te Kāwanatanga. Ko Ngā Tama Toa rāua ko Te Reo Māori Society ngā rōpū nā rātou tētahi petihana i kawe hei whakahoki i te reo Māori ki te marau kura (Te Rito, 2008). He hononga nui ō ēnei rōpū e rua ki ngā whare wānanga Pākehā. Ko ètahi he ākonga nā Ahorangi Ranginui Walker, ko ètahi he 'tauira ture', ko te katoa i mōhio kāore i te tika te mahi a te pūnaha Pākehā ki te Māori. Ko ngā mātāwaka o ngā rōpū, he manene ki ngā tāone nui, à, ko te nuinga o ngā tāngata ehara i te tangata kōrero Māori (Te Rito, 2008). Nā te āhua o tēnei noho tawhiti i te ūkaipō, me te māramatanga ki ngā pānga o te mātauranga ki tēnei mea te noho herekore me te noho rawakore, ka mārama kehokeho rātou ki te hanganga tōrangapū o te oranga Māori. I ēnei rā, ka kīia he tino 'oho' (i roto i te reo Pākehā ko te 'woke' te kupu a te rangatahi, kia noho mataara i te ao) ēnei momo, ahakoa i noho rātou hei rāwaho i te nuinga o ngā hapori Pākehā me ngā hapori Māori. He tāngata mōhio ki ngā mahi taihara a te Pākehā, he tāngata mārama ki ngā hua me ngā pānga o èrā mahi taihara ki te aupēhi i te oranga o te Māori. He tāngata kua hūneinei ki te whakawhitinga o te Māori i te iwi rangatira ki te iwi pararau. He tāngata e hiahia ana kia 'whakarerea ngā kaka taumaha, kia piki te ihirangaranga'.

Kāti, ko te hua o te petihana, ko te 'Rā Whakanui i te Reo Māori’ ki roto i ngā akomanga puta noa i te motu (ko te Māori Language Day tērā). Ko tā Pāpā Pita Sharples ki te motu:

Kei te maumaharatia tonutia te Rā o te Reo Māori ia tau, ia tau, i te rā tekau mā whā o Mahuru. Ko te rā tèrā o te tau 1972, i whakatakotoria ai tētahi tono ki mua i te aroaro o te Pāremata. Toru tekau mano ngā tāngata i tāmoko i te tono, kia akona te reo Māori i roto i ngā kura o te motu (Sharples, 2010)

I te tau 1975 , ka huri taua rā ki Te Wiki o te Reo Māori. Nō te tau 1980, i kitea i te mate haere te reo toi o te Māori (Benton, 1991). I mautohe tonu te Māori, kāti, i puta ētahi kaupapa reo Māori ki te whakarauora $\mathrm{i}$ te reo o te motu.

Ko te mātāmua o ngā kaupapa ko Whakatupuranga Rua Mano, he rautaki mō te whakatupu i te reo Māori me ōna tikanga, me ngā wawata o ngā iwi (Winiata, 2006). Ko Ngāti Raukawa (Te Au ki te Tonga) rātou ko Te Āti Awa, ko Ngāti Toa ngā kaihāpai i te kaupapa o Whakatupuranga Rua Mano. Ko te hua o tēnei rautaki ko Te Wānanga o Raukawa i te tau 1981. Kāore te Kāwana i tautoko i tēnei whare wānanga Māori, nō reira, nā ēnei iwi anō te wānanga i utu (Warren, 2017). Kotahi tau whai i muri mai, ka whakatūria te Kōhanga Reo. Nā ngā hapori anō ērā i utu, i tautoko ki te moni, i whakarauemi (Tomlins-Jahnke \& Warren, 2011). Ko ngā 
kaumātua ngā kaiako, ko ngā whānau ngā ringa tautoko. Ko te whāngai i te reo Māori me ōna tikanga te pūtake o te Kōhanga Reo. I reira ka kitea anō te whakaahua mai o te wairua Māori me te hiahia kia 'Māori' te whakatupu i ngā tamariki Māori. Mō ngā tau 168 kua uru mai ngā àhuatanga o te whakaako Pākehā hei huarahi matua mō ngā tamariki Māori. Ko taua huarahi, he huarahi e mea ana ki te Māori e mōhio ana te Pākehā ki ngā painga mō te Māori. Engari ko te whakatū Kōhanga Reo, he tohu e whakapono ana te Māori ki tōna ake tuakiri, ki tōna ahurea ako, $\bar{a}$, e hiahia ana te Māori ki te whakaora i à tātou tikanga kua roa e tāmia ana, ki te whakanau anō hoki i ngā huarahi o te Pākehā. I roto i te 20 tau kua whakatūria ngā kōhanga Reo e 704 (Reedy, 2000).

Ki te tautoko atu i ngā whānau kua roa e noho makuhea ana ki te reo Māori, i whakatūria Te Ātaarangi hei kaupapa whakarauora i te reo Māori i waenga i ngā pakeke. Ko tēnei kaupapa e rite ana ki ètahi atu kaupapa whakarauora reo ki tāwāhi (titiro ki Gattegno, 1963). Nā te whakapakeke tamariki i te reo Māori ki te Kōhanga Reo, me whai kura tuatahi aua tamariki. Nō te tau 1985, ka tuwhera te Kura Kaupapa Māori (Ministry of Education, 2010). Ahakoa kāore he pūtea tautoko a te Kāwana, i kōkiri tonu te whānau kia anga whakamua tēnei kaupapa. He hokinga mahara tēnei ki te Education Act 1877—nā te Māori anō ona kura i tautoko ki te tahua. Kāti, ahakoa ko te reo Māori te puna o te Kōhanga Reo me te Kura Kaupapa Māori, kua roa te Māori e mārama ana ki te aupēhi a ngā kura Pākehā ki ngā tamariki Māori, me te patu i te wairua Māori. Kāore i tino kitea te angitu o te Māori i roto i ngā kura 'aunoa', i ngā kura Pākehā o te motu. E ai ki a Rawiri Wright, he tai tapu te auraki mō te hekenga mai o ngā waka i Hawaiki ki Aotearoa. Ko tērā te 'au' matua. Nā te mea kei ngā kura Pākehā te nuinga o ngā tamariki, i tapaina atu ko te kura auraki tērā, arā, ko te kura matua. Engari ehara ērā kura i te kura tapu mō ngā tamariki Māori. Nō reira, ko tā Rawiri atu ki ngā tauira o Te Aho Tātairangi me Te Aho Paerewa (ki tētahi wānanga i te tau 2018), me tapa kē te 'aunoa' ki ngā kura Pākehā. Kāti, he waka kawe oranga wairua te Kōhanga Reo me te Kura Kaupapa Māori.

Nō reira, he aha te take o te ahunga mai o te Kōhanga Reo me te Kura Kaupapa Māori?

1. I te mate haere te reo Māori, nā reira me whakarauora ake te reo.

2. Kua roa te Māori e tāmia ana ki ngā āhuatanga o te Pākehā.
3. Ko te tuakiri o te Māori, i whakahāwinitia ki te whakahiranga o ngā āhuatanga Pākehā.

4. Me noho pū te ao Māori mō ngā tamariki.

5. I te rahua te Māori ki ngā kura aunoa. Me angitu kē ngā tamariki ki roto i ngā kura.

Ka nui te amowheke o ngā mātua ki te Kāwana i te kore tonu o ngā ākonga Māori e tino eke i ngā taumata i te kura (Tocker, 2015). I mōhio pū ngā mātua Māori, ka kore e hē kē atu à rātou whāinga i ngā Kura Kaupapa Māori i ērā a te Kāwana (Tocker, 2015). Mā te aha i te kore. Ka mutu, he mautohe anō te whakatūria o ngā Kura Kaupapa Māori hei 'tauranga ātete' i ngā tau aupēhi. Otirā, kua roa ngā āhuatanga Māori e noho ana ki 'waho o te manawa'. Kua pēhia te wairua o te Māori, à, kua whākorekore te tuakiri Māori. Kua eke ki te wā me manawanui te Māori, me manawa ora, me tū mai te manawa Māori hei oranga mō ngāi tātou.

Nō taua tau anō, nō te tau 1985 i tukuna te Kerēme mō Te Reo Māori ki te Rōpū Whakamana i te Tiriti o Waitangi (New Zealand Waitangi Tribunal, 1986) e Huirangi Waikerepuru me Ngā Kaiwhakapūmau i te Reo.

E ai ki a Campbell rāua ko Stewart (2009), e rua ngā take o te Kura Kaupapa Māori. Ko te tuatahi, ko te whakarauora i te reo Māori. Ko te tuarua, ki te puretumu i te rahua o te Māori ki ngā kura, ki te taha mātauranga. Ka hono atu ērā momo rahua ki te mātauranga, te whakawhiti ki te whare wānanga, te whai tūranga mahi, te whakauru ki ngā kaupapa tōrangapū, me te noho hei kirirarau ki Aotearoa, ki te ao whānui anō hoki (Campbell \& Stewart, 2009). Ko te whakaaro ia, mā te reo te tuakiri e ora ai, mā te tuakiri ora te tangata e ora ai.

E tika ana ngā kupu a Rawiri Wright (2009) . . .

Whakarerea ngā kaka taumaha

Kia piki te ihirangaranga e

Kia tiaho mai te wairua ...

Te Aho Matua, Kura Kaupapa Māori

Hei waka kawe oranga wairua

Hei korowai i ngā awhero hira e...

Mānawa mai rā ko Te Aho Matua! Ahakoa kua tāwēwē te aho Māori i roto i ngā tau, mā Te Aho Matua te ahurea Māori me ōna āhuatanga katoa e renarena ki te aho tapu o te Māori. Me kaua e whakaaro he ariā noa iho Te Aho Matua. I te mutunga iho, he rongoa kē Te Aho Matua. Mā Te Aho Matua e tapuhi te wairua o ngā tamariki me ō rātou whānau. I titongia Te Aho Matua e Katerina Mataira mā (Tocker, 2015), ā, i puta ki te 
Education (Te Aho Matua) Amendment Act 1999 (S155a). Mā ngā wāhanga e ono o Te Aho Matua e tino kitea ai tōna whāinga me ōna wawata mō ngā tamariki. Hei tauira, ko te wāhanga tuatahi o Te Aho Matua, ko Te Ira Tangata. E rua ngā kōrero e poipoi ana i te wāhanga tuatahi:

- Ahakoa iti. He iti māpibi pounamu.

- He kākano i ruia mai i Rangiātea. E kore ia e ngaro.

Kua hīkoko te Māori ki ngā āhuatanga o Te Aho Matua i ngā kura aunoa i roto i ngā tau. Ko ngā tatauranga mō te rahua o te Māori i te ao mātauranga te taunaki (Warren, 2013).

Nā Te Aho Matua me ōna whāinga ngā āhuatanga Māori i whakahoki ki te kuku o te manawa. He tauranga ātete tēnei mahi hei whakaatu ki te ao 'he Māori ahau, he wairua Māori, he whakapono Māori, he ahurea Māori, he mōhiotanga Māori, he mātauranga Māori e kore e ngaro'. Ka whāia ēnei āhuatanga, nā te mea kei te mōhiotia he aha te huarahi pai hei whai-ko te ao Māori tērā. Ka pērā i ngā kōrero a Ahorangi Linda Tuhiwai-Smith (1999), me whakahoki ngā mea Māori katoa ki te pokapū o te oranga Māori.

Heoti, ahakoa kāore te Kāwana i tautoko i te Kura Kaupapa Māori, nō te tau 1999, ka puta Te Aho Matua hei ture ki roto i te Education (Te Aho Matua) Amendment Act (S155a). He tohu tēnei kua tahuri mai te Kāwana ki te tūtohu i te huarahi whakaako Māori. E whakaatu ana hoki tēnei i te māia o ngā pou whakarae e kawe ana i te kaupapa-he ururoa. Me mihi atu ki ngā mātua o te Kura Kaupapa Māori, o Te Aho Matua; ki a Pāpā Pita Sharples, a Whaea Tuki Nepe, a Whaea Katerina Mataira, a Linda Smith, a Hingangaroa Smith, a Rawiri Wright, a Kathy Dewes, a Toni Waho, a wai atu. Nā koutou tēnei kaupapa i āki ki tōna angitu.

Nō reira, he aha ngā hua o te Kōhanga Reo me te Kura Kaupapa Māori? He nui ngā kōrero a Whaea Nuki Tākao mā (2010) e pā ana ki ngā hua o te Kura Kaupapa Māori. Engari, kei konei ētahi kōrero whakarāpopoto i ngā hua:

a) He tamariki kōrero Māori

i. E kōrero Māori ana te $5 \%$ o ngā tamariki Māori (Statistics New Zealand, 2013). He rite ki te tokomaha o ngā tamariki ki ngā Kura Kaupapa Māori (Statistics New Zealand, 2013). Nō reira, e āhei ana pea te kī, ki te kore te Kura Kaupapa Māori, kua kore he tamariki kōrero Māori. He tohu hoki tēnei, mā ngā kura ngā tamariki (me ō rātou whānau) e akiaki ki te kōrero Māori. ii. He hapori kōrero Māori. E ai ki a Joshua Fishman (1991), mā te hapori te reo e tuku iho, e whakarauora.

e) He tamariki tuakiri ora

i. He tamariki e ako ana i Te Aho Matua. Kei te mārama ki: Te Ira Tangata me te tapu o te tangata; Te Reo me te tapu o ngā reo katoa; Ngā Iwi, tōna whakapapa me ōna hononga katoa; Te Ao, kia tupu te tamaiti hei tamariki o te ao whānui; Āhuatanga Ako me te hari koa o te ako mā ngā huarahi maha (kaumātua, ngū, raweke, tuakana, te mea te mea); Te Tino Uaratanga me te tipu o ngā āhuatanga Māori o te tamaiti "kia tū hei raukura mō tōna iwi” (Education (Te Aho Matua) Amendment Act 1999, S155a)

i) He kaiako atawhai

i. Me poipoi ngā kaiako i ngā āhuatanga katoa o Te Aho Matua. Ka mārama te kaiako ki te tapu o te tamaiti me ōna hononga ki Rangiātea.

o) He tamariki whai tohu

i. Ka eke te taiohi wharekura ki ngā taumata e tika ana mō te whai tohu NCEA (National Certificate of Educational Achievement). Heoi anō, he angitu rawa te tauira wharekura i roto i ngā tatauranga mātauranga o te motu (NZME Educational Media, 2019, Warren, 2013).

ii. Ko te nuinga o ngā tamariki Māori he tamariki o te kura aunoa, engari kāore rātou i te ora ki te whiwhinga tohu NCEA.

u) Te kore puta ki te aroaro o te ture (Ministry of Justice)

i. Ko te nuinga o ngā tāngata kua whakaaetia e te ture kua taihara, he Māori (Policy, Strategy and Research Group, Department of Corrections, 2007). Engari, e mokorea ana te kite i te tamaiti nō te kura kaupapa Māori i te aroaro o te ture (Annette Sykes, kōrero ā-waha, 5 Pepuere 2018). Ehara i te mea ka kore ngā tamariki kura kaupapa Māori e hara i raro i te ture, engari he tokoiti tonu rātou. E tohu ana tēnei i te kaha poipoi a Te Aho Matua, a te Kura Kaupapa Māori, me ngā whānau, i ngā tamariki mokopuna.

Ko te tino hua o te kura kaupapa Māori ko te rongo me te mārama ki ngā tikanga Māori pēnei i te manaakitanga, $i$ te whanaungatanga, i te noho tuakana/teina. E ai ki ngā raukura, ko èrā ngā tino taonga e puritia rawatia ana whai muri i te wehenga i te kura (Tākao mā, 2010). Arā, nā te Kura Kaupapa Māori i puta ai te tamaiti: 
Hei korowai i ngà awhero hira e...

Kia puawai mai hei matataki e

Hei matahiapo, hei mataaho

Mō te motu whānui (Wright, 2009)

\section{Ariā Kaupapa Māori}

Hei waka kawe oranga wairua (Wright, 2009)

Ko te Kaupapa Māori he ariā, he huarahi rangahau anō hoki (Tuhiwai-Smith, 1999; Pihama, 2015). I hōrapa te āhuatanga o te tāmi i te Māori. I tino kitea èrā āhuatanga ki ngā whare wānanga anō hoki; me te tinei i te mōhiotanga me te mātauranga Māori. He ariā ātete tēnei kia whai wāhi te Māori me ōna whakaaro ki roto i te ao mātauranga aunoa. He ariā e mārama ana ki te hononga o te mahi rangahau ki te tāmitanga. He mahi motuhake ēnei momo kōrero kia mārama pai tātou ki ngā tini āhuatanga o te ao e here nei i a tātou. Ko ètahi o ngā hononga: ko te 'hegemony' te ritenga o ngā tikanga ki ngā tikanga Pākehā (te iwi tāmi rānei) (Gramsci, 1971); ko te 'pedagogy of the oppressed' ngā tikanga ako mō te tāmi tangata (Freire, 1972). Anei ngā kōrero a Tuhiwai-Smith (1999):

Under colonialism indigenous peoples have struggled against a Western view of history and yet been complicit with that view. We have often allowed our 'histories' to be told and have then become outsiders as we heard them being retold. Schooling is directly implicated in this process. Through the curriculum and its underlying theory of knowledge, early schools redefined the world and where indigenous peoples were positioned within the world. From being direct descendants of sky and earth parents, Christianity positioned some of us as higher-order savages who deserved salvation in order that we could become children of God. (Tuhiwai-Smith, 1999, wh. 33).

He ariā matua te ariā Kaupapa Māori mā te tauira e rangahau ana i te whare wānanga. Ka kitea i te kōrero a Tuhiwai-Smith he hononga nui kei waenga i te tāmi, te rangahau, me te marau. Me maumahara tātou ki te hononga o te rangahau ki te kura me te marau hei tauranga ātete, hei huarahi whakarauora i ngā mōhiotanga Māori me ngā kōrero tuku iho.

\section{Ngā tuhinga reo Māori}

Kia puawai mai hei matataki e (Wright, 2009).
I te tuatahi, ko te tikanga o tēnei tuhinga, me aroā te iti o ngā tuhinga reo Māori e pā ana ki Te Aho Matua (Cashell-Warren, 2019) me ngā āhuatanga tōrangapū e hāngai ana ki te Kura Kaupapa Māori. Heoi anō, i te aromatawainga o ngā kōrero, i kitea he āputa nui mō te ahunga o te Kura Kaupapa Māori. Nō reira, kua aro kē tēnei tuhinga ki te ahunga mai o te Kura Kaupapa Māori. Tēnā, me whakarāpopoto te nawe ki konei.

Ko te nuinga o ngā tuhinga i te reo Māori, he tuhinga o mua (pērā i ngā niupepa Māori, ngā Meneti Kōti Whenua Māori me ētahi atu kōrero a ngā tūpuna), he pakiwaitara, he kōrero mō te reo Māori rānei. Ehara i te mea kua hōrapa whānui te reo Māori i ngā marau maha (Stewart, 2019). Ko te ngako o te nawe, kāore anō ngā kōrero tōrangapū kia puta i te reo Māori, kia ngāwari ai te pānui me te whai māramatanga ki aua take. Kāore anō ngā kōrero mō ngā marau maha kia puta. I roto i ngā tau kua mātua aroā i a tātou te whakarauoratanga o tō tātou reo Māori. Iènei tau, kua whāwhai te whakaputa i ngā rauemi kei te reo Māori. Kua tae tātou ki te wā kia puta te mahi a te rauemi reo Māori mō ngā kaupapa huhua e pā ana ki te ao Māori.

Heoi anō, ehara i te mea mā te pānui tuhituhi anake e mārama ai. Kua puta pea aua kōrero i roto i ngā waiata, i ngā mōteatea hei kōrero tuku iho. Nō reira, me whai rautaki tātou mō te ruku ki aua māramatanga, ka tahi. Ka rua, me tahuri te ao whare wānanga ki te whakanui me te pāhekoheko i aua māramatanga ki ngā mahi whare wānanga. Ko te take ia, ka puta te raukura ki te whare wānanga, kei whea ōna anō rauemi reo Māori? Me pēhea ia e mārama ai ki ngā kōrero mautohe mō te Kaupapa Māori? Me whai rautaki anō tātou ki te poipoi i te reo Māori ki ēnei taumata, engari me kounga tonu te mahi rangahau ki tēnei taumata. Kāti, me kaha te whakaputa i ngā kōrero i roto i te reo Māori mō ngā kaupapa katoa.

Ehara i te mea he kounga noa i te mea i tuhia i te reo Māori. He nui tonu ngā tuhinga ngoikore i te reo Pākehā. Nā reira, me kairangi tonu ngā kai o roto. E akiaki ana a Smith (2000) i a tātou kia mārama tātou ki ngā momo ariā katoa hei tautoko i à tātou mahi wewete.

\section{Hei whakakapi}

Hei matahiapo, hei mataaho

Mō te motu whānui (Wright, 2009).

Ahakoa kua tāmia te iwi Māori e te taiāniwhaniwha mai o te manene Pākehā, kua mautohe tonu te Māori. Ko te Kōhanga Reo me te Kura Kaupapa 
Māori ètahi o ngā kaupapa ātete i puta i roto i ngā tau tata nei. Ko te whāinga o tēnei tuhinga, ko te tuku i ètahi kōrero reo Māori e pā ana ki te whakapapa tōrangapū o te Kura Kaupapa Māori. I te tuatahi, i aroā te pānga o ngā ture Pākehā me te kura ki te Māori. He wāhi taimaha i tino herea ai te Māori (Tuhiwai-Smith, 1999). I mātaia anō hoki ko ngā mahi ātete a te iwi Māori. I te tuarua, i aroā te ahunga mai o te Kura Kaupapa Māori hei tauranga àtete, hei waka kawe oranga wairua mō te Māori. Arā, i tirohia ngā take o te Kura Kaupapa Māori me te tini hua o te kaupapa. Ka toru, he tono tēnei tuhinga kia:

a) puta ētahi tuhinga kounga i te reo Māori, e pā ana ki ngā ariā hei arataki i te whakaaro o ngā tauira whare wānanga,

e) whai rautaki anō ngā whare wānanga kia pāhekoheko ngā kōrero o mua, kia puta te tuhinga taiea mā ngā tauira.

Ka mutu ko te raukura te matahiapo, ko rātou hoki ngā toa taua mō te reo Māori. Nō te wā o Tangaroa i te marama o Paengawhāwhā i te tau 2019, i hui atu ngā raukura o te motu ki Ruamatā. Ko te kaupapa matua, he aha tā rātou hei koha atu ki Te Aho Matua (Pewhairangi, 2019). Ko ngā kōrero matua i puta, me pēwhea mātou ngā raukura e tautoko ai i te hunga kātahi anō ka puta i te wharekura, me pēwhea hoki e koke whakamua ai ngā kura kaupapa Māori. Me wānanga tonu tēnei kaupapa.

Kāti, ka hoki ki ngā kōrero a Pāpā Rawiri Wright hei whakakapi, hei whakarāpopoto i te tuhinga nei me ngā kupu whakanui i te Kura Kaupapa Māori.

Tēnei au te ira nō tuawhakarere,

Ko te ira Atua, tēnei au

He konehunehu nō te kore, nō te pō,

Nō te marae ātea, āio [ā-Io]

Ko te ira Atua, ko te ira wahine

Ko te ira tāne, tēnei au

Ka kōtui ngātahi ki te ira tangata

Tau mai rā ki te papa tipua

Tōiri ana mai ko te ngao rongomau

Nō te ohonga mai a Io

Whakarerea ngā kaka taumaha

Kia piki te ihirangaranga e

Kia tiaho mai te wairua ...

Te Abo Matua, Kura Kaupapa Māori

Hei waka kawe oranga wairua

Hei korowai i ngā awhero hira e...

Kia puawai mai hei matataki e

Hei matahiapo, hei mataaho
Mō te motu whānui

Tēnei au te ira Atua, tēnei au te ira Atua,

Tēnei au te ira Atua e (Wright, 2009).

*E tika ana anō kia rere a mihi ki ngā tumu whakarae. Koia ēnei, ko Pāpā Pita Sharples, ko Kōkā Katerina Mataira, ko Aunty Cathy Dewes, ko Pāpā Graham Smith, ko Kōkā Linda Smith, ko Whaea Tuki Nepe, ko Whaea Rahera Shortland, ko Pāpā Pem Bird, ko Pāpā Toni Waho, ko Pāpā Rawiri Wright, ko Waene Renee Wright, ko koutou katoa. Me mihi hoki ki ngā whānau o ngā kura kaupapa Māori-kāore e ārikarika ngā mihi ki a koutou katoa. Tēnā koutou katoa.

\section{Rārangi pukapuka}

Apple, M. (1996). Cultural politics \& education. New York, NY: Teachers College, Colombia University.

Barrington, J. M., \& Beaglehole, T. H. (1974). Māori schools in a changing society: An historical review. Wellington, NZ: New Zealand Council for Educational Research.

Benton, R. A. (1972). Hewaka pakaru kino? Wellington, NZ: New Zealand Council for Educational Research.

Benton, R. A. (1991). Māori English: A New Zealand myth. In J. Cheshire (ed.). English around the world: Sociolinguistic perspectives (wh. 187-199). Cambridge, UK: Cambridge University Press.

Campbell, R., \& Stewart, G. (2009). Ngā wawata o ngà whānau Wharekura. Aspiration of whānau in Māori medium secondary schools. Wellington, NZ: New Zealand Council for Educatiōnal Research.

Cashell-Warren, KT. B. (2019). Te Abo Matua: he rārangi pukapuka o ngā tuhinga me ngā kōrero. Kāore anō kia taa. Te Papaioea: Te Kunenga ki Purehuroa (Massey University).

EducationCentral.co.nz (23 April, 2018). NCEA results: see how your school rates. I tikina i: https://educa tioncentral.co.nz/_trashed-3/

Fishman, J. A. (1991). Reversing language shift: Theory and practice of assistance to threatened languages. Clevedon, UK: Multilingual Matters.

Fournier, S., \& Crey, E. (1997). Stolen from our embrace: The abduction of First Nations children and the restoration of aboriginal communities. Vancouver, Canada: Douglas and McIntyre.

Freire, P. (1970). Pedagogy of the oppressed. New York, NY: Seabury.

Gattegno, C. (1963). Teaching foreign languages in schools: The silent way (1st ed.). Reading, UK: Educational Explorers.

Gramsci, A. (1971). Selections from the prison notebooks. (Edited and translated by Quitin Hoare and Geoffry Smith). London, UK: Lawrence and Wishart.

Ihimaera, W., \& Kelly, H. (2017). Sleeps standing Moet $\bar{u}$. Auckland, NZ: Penguin Random House.

King, M. (1977). Te Puea: A biography. Auckland, NZ: Hodder and Stoughton. 
King, M. (1988). Apirana Ngata: e tipu e rea. Wellington, NZ: Department of Education.

Mataamua, R. (2017). Matariki: te whetu tapu o te tau. Wellington, NZ: Huia Publishers.

Ministry of Education. (2010). Ngā whanaketanga rumaki Māori. I tikina i: www.education.govt.nz/

National Advisory Committee on Māori Education. (1970). Māori education: Report of the National Advisory Committee on Mãori Education. Wellington, NZ: Department of Education.

New Zealand Qualifications Authority. (11 April 2018). 2017 NCEA and New Zealand Scholarship attainment data available. New Zealand Qualifications Authority. I tikina i: https://www. nzqa.govt.nz/about-us/news/2017-nceanzscholar shipdata-available/

New Zealand Waitangi Tribunal. (1986). Report of the Waitangi Tribunal on the te reo Māori claim (Wai 11). Wellington, NZ: Author.

NZME Educational Media. (2019). NCEA results: See how your school rates. I tikina i: https://education central.co.nz/_trashed-3/

Papps, T. (1985). Growth and distribution of population. In Population of New Zealand / Economic and Social Commission for Asia and the Pacific 12, Vol. 1. New York, NY: United Nations.

Pewhairangi, T. A. (Paraire 26, 2019). "Kura kaupapa Māori hold reunion to reflect on Te Aho Matua." Te Karere. I kitea i https://www.facebook. com/tekareremaorinews/videos/1263582797150 $636 / ? \mathrm{t}=4$

Pihama, L. (2015). "Kaupapa Māori theory: Transforming theory in Aotearoa", In L. Pihama, S-J. Tiakiwai \& K. Southey (eds.). Kaupapa Rangahau: a reader. A collection of readings from the Kaupapa Rangahau Workshop Series. (wh. 5-14). Hamilton, NZ: Te Kotahi Research Institute.

Policy, Strategy and Research Group, Department of Corrections. (2007). Over-representation of Māori in the criminal justice system: An exploratory report (September 2007). Wellington, NZ: Department of Corrections. I tikina ki https://www.correc tions.govt.nz/_data/assets/pdf_file/0004/672574/ Over-representation-of-Māori-in-the-criminaljustice-system.pdf

Pool, D. I. (1991). Te iwi Māori: A New Zealand population, past, present \& projected. Auckland, NZ: Auckland University Press.

Poutu, H. N. (2014). Kia tiori ngā pīpi: ma te aha e kōrero Māori ai ngā taitamariki ngā wharekura o Te Aho Matua? He tuhinga roa hei whakatutuki i ngā tikanga o Te Tohu Kairangi. Te Kunenga ki Pūrehuroa, Te Papaioea, Aotearoa.

Reedy, T. (2000). Te reo Māori: the past 20 years and looking forward. Oceanic Linguistics, 39(1), 157-169.

Sharples, P. (1994). Kura Kaupapa Māori. In H. McQueen (Ed.), Education is Change (wh. 11-21). Wellington, NZ: Bridget Williams Books.

Sharples, P. (30 July, 2010). "Speech to iwi and community stakeholders." Beehive.govt.nz. I tikina i: https://www.beehive.govt.nz/speech/ speech-iwi-and-community-stakeholders
Simon, J. A., Smith, L. T., \& Cram, F. (2001). A civilising mission? Perceptions and representations of the Native Schools system. Auckland, NZ: Auckland University Press.

Smith, G. H. (2000). Protecting and respecting indigenous knowledge. In M. Battiste (Ed.). Reclaiming Indigenous voice and vision. (wh. 209-224). Vancouver, Canada: UBC Press.

Statistics New Zealand. (2013). Statistics New Zealand. Speakers of te reo Māori. New Zealand Progress Indicators. I tikina i: http://www.stats.govt.nz/ browse_for_stats/snapshots-ofnz/nz-progress-indi cators/home/social/speakers-of-te-reo-Māori.aspx

Stewart, G. H. (2019). "He iti pounamu.” MAI Journal, 8(1), 77-90. I tikina i: http://www.journal.mai. ac.nz/sites/default/files/FINAL\%20MAIJrnl_8_1_ Stewart_02.pdf

Tākao, N., Grennell, D., McKegg, K., \& Wehipeihana, N. (2010). Te piko o te māhuri: the key attributes of successful Kura Kaupapa Māori. Wellington, NZ: Ministry of Education.

Te Rito, J. S. (2008). Struggle for the Māori language: he whawhai mō te reo Māori. MAI Review, 2(6), 1-8. I tikina i http://ojs.review.mai.ac.nz/index. php/MR/article/view/164/169

The New Zealand Digital Library. (n.d.). Niupepa: Māori newspapers. [he whārangi ipurangi] I tikina i: http://www.nzdl.org/gsdlmod?a=p\&p=about\&c $=$ niupepa\&l=mi\&nw=utf -8

Tocker, K. (2015). The Origins of Kura Kaupapa Māori. New Zealand Journal of Educational Studies, 50(1), 23-38. 10.1007/s40841-015-0006-z, http:// hdl.handle.net/2292/27917

Tomlins-Jahnke, H. (2006). The place of cultural standards in indigenous education. MAI Review, 1(1), 1-11. I tikina atu ki: http://review.mai.ac.nz/MR/ article/download/100/100-540-1-PB.pdf

Tomlins-Jahnke, H., \& Warren, K.T. (2011). Full, exclusive and undisturbed possession: Māori education and the Treaty. In V.M.H. Tawhai \& K. Gray-Sharp (Eds.). Always Speaking: The Treaty of Waitangi and public policy (wh. 21-33). Wellington, NZ: Huia Publishers.

Tuhiwai-Smith, L. (1999). Decolonizing methodologies: Research and indigenous peoples. Dunedin, NZ: University of Otago Press.

Von Dadelszen, E.J. (1901). Results of a census of the colony of New Zealand taken for the night of the 31st March, 1901. [Digitised collection]. Statistics New Zealand. I tikina atu i: http://www.stats.govt. nz/browse_for_stats/snapshots-of-nz/digitised-col lections/census-collection.aspx

Walker, R. (1984). The genesis of Māori activism. The Journal of the Polynesian Society, 93(3), 267-281. I tikina i: http://www.jstor.org/stable/20705873

Walker, R. (1990). Ngā pepa a Ranginui: The Walker Papers. Auckland, NZ: Penguin Books.

Walker, R. (2016). Reclaiming Māori education. In J. Hutchings \& J. Lee-Morgan (Eds.), Decolonisation in Aotearoa: Education, research and practice (wh. 19-38). Wellington, NZ: New Zealand Council for Educational Research.

Warren, K. F. (2013). The legacy of Māori education—a 
view in 2013. In T. Black, C. Cunningham, B. Jahnke, H. Tomlins-Jahnke, \& T.K. Kingi (Eds.), Matariki: a monograph prepared by Te Mata o te Tau The Academy for Māori Research and Scholarship. (wh. 15-31). Palmerston North: Te Mata o te Tau (The Academy for Māori Research and Scholarship). Warren, K. T. (2017). Te reo Mãori: Analysing the colonial history of the native Māori language of Aotearoa (New Zealand). Revista LinguiStical Revista do Programa de Pos-Graduacao em Linguistica da Universidade Federal do Rio de Janeiro, 13(1), 349-366. ISSN 2238-975X 1. https://revistas.ufrj.br/index.php/rl
Winiata, P. (2006). The role of tikanga Māori institutions in the protecting, sustaining and nurturing of traditional knowledge. In J.S. Te Rito (Ed.), Proceedings of the Mátauranga Taketake: Traditional Knowledge Conference Indigenous Indicators of Well-being: Perspectives, Practices, Solutions (wh. 199-212). Auckland, NZ: Ngā Pae o te Māramatanga.

Wright, R. (2009). Tēnei au. Tēnei Au [Kōpae Waiata]. Rotorua: Te Whānau o Ruamata. 